\title{
Miranda
}

Revue pluridisciplinaire du monde anglophone /

Multidisciplinary peer-reviewed journal on the English-

speaking world

$17 \mid 2018$

Paysages et héritages de David Bowie

\section{La construction identitaire dans le cinéma de Ngozi Onwurah : (re-)stylisation du corps et stratégies de subversion}

\section{Émilie Herbert}

\section{(2) OpenEdition}

\section{Journals}

Édition électronique

URL : http://journals.openedition.org/miranda/13505

DOI : $10.4000 /$ miranda. 13505

ISSN : 2108-6559

\section{Éditeur}

Université Toulouse - Jean Jaurès

\section{Référence électronique}

Émilie Herbert, «La construction identitaire dans le cinéma de Ngozi Onwurah : (re-)stylisation du corps et stratégies de subversion », Miranda [En ligne], 17 | 2018, mis en ligne le 21 septembre 2018 consulté le 16 février 2021. URL : http://journals.openedition.org/miranda/13505; DOI : https:// doi.org/10.4000/miranda.13505

Ce document a été généré automatiquement le 16 février 2021.

\section{c) (1)}

Miranda is licensed under a Creative Commons Attribution-NonCommercial-NoDerivatives 4.0 International License. 


\title{
La construction identitaire dans le cinéma de Ngozi Onwurah : (re-)stylisation du corps et stratégies de subversion
}

\author{
Émilie Herbert
}

En janvier 2018, le London Short Film Festival proposait une courte rétrospective de l'œuvre d'une «pionnière du cinéma Black-British $»^{1}$, la réalisatrice britanniconigériane Ngozi Onwurah. Trois des courts-métrages les plus influents de la réalisatrice y étaient projetés : Coffee Coloured Children (1988), The Body Beautiful (1991) et The Flight of the Swan (1992). CEuvres incontournables au sein de sa filmographie, ces trois courtsmétrages ont également pour point commun d'évoquer la problématique de la construction identitaire des enfants noirs et métis. Largement inspirés de souvenirs autobiographiques, les personnages d'enfants chez Onwurah sont constamment présentés au cœur d'un parcours initiatique durant lequel leur identité raciale se construit. Ce parcours est dépeint comme conflictuel puisque les discours racistes auxquels ces jeunes protagonistes sont confrontés - et leur internalisation de ces discours - provoquent différents effets psychiques (déni identitaire, haine de soi, mélancolie) et corporels (violence physique, autopunition) négatifs. Les films présentent cependant des stratégies de subversion qui visent à créer de nouvelles formes d'identification : la fin de l'enfance et le passage à l'adolescence s'avèrent être une étape cruciale pour ces jeunes héro.ïne.s, qui se défont des carcans racistes à travers différents rituels de résistance re-stylisant le corps. Les concepts de stylisation du corps et de performativité, issus des travaux de la philosophe américaine Judith Butler $(1990,1993)$, formeront le cadre de départ de notre analyse du cinéma de Ngozi Onwurah. Nous verrons de quelle manière une approche phénoménologique et une perspective postcoloniale permettent de compléter les théories butlériennes dans l'élaboration d'une performativité raciale centrée sur le corps racisé. L'étude de l'affirmation d'une identité noire à travers la re-signification des discours et des comportements associés à la blackness ${ }^{2}$ - un processus que la sociologue Shirley Tate 
nomme, en référence à Frantz Fanon, «Peau Noire/Masque Noir» (Tate, 2005) - nous permettra d'analyser les stratégies représentationnelles employées par la cinéaste pour subvertir les processus normatifs imposés sur des corps qui sont chez elle « le paysage central d'un discours anti-impérialiste » (Foster, 1997, 7).

\section{Les travaux de Judith Butler : Performativité du genre et stylisation du corps}

2 Dans sa théorie de la performativité du genre, Butler contredit l'idée essentialiste selon laquelle chaque être humain posséderait dans son essence profonde un genre inné, naturel et ontologique. Pour la philosophe, c'est au contraire l'incorporation de normes sociales, à travers une réitération et une répétition indéfinie d'énoncés récités par les corps, qui crée le genre. C'est cette réitération que Butler nomme la «stylisation du corps »: «L'effet du genre est produit par la stylisation du corps et doit donc être compris comme la façon banale dont toutes sortes de gestes, de mouvements et de styles corporels donnent l'illusion d'un soi genré durable» (Butler, 2005, 265). En répétant cette stylisation de leurs corps, hommes et femmes développent donc des comportements, des capacités ou des aptitudes différents, et à travers un cycle de routines qui se transforme en une seconde nature, le genre devient un acte performatif. En linguistique, une parole est dite performative quand elle ne se contente pas d'être un simple discours, mais qu'elle provoque la réalisation d'un acte concret: lorsque le juge condamne le prisonnier ou que le maire officialise un mariage, leurs mots ( $\mathrm{Je}$ vous condamne » ou "Je vous déclare mariés»), résultent en une action concrète. À partir de leur position de pouvoir, leur parole devient donc performative ${ }^{3}$. De la même manière, Butler propose l'idée que le performatif produit, et agit sur, ce qu'il nomme. Pour la philosophe, le genre devient performatif grâce à la répétition compulsive des normes, car c'est à travers les pratiques quotidiennes du genre - par exemple pour une femme en se maquillant, en portant des vêtements féminins ou des talons hauts - que notre identité genrée se forme. Cette dernière n'est pas donc pas naturelle, fixe et stable mais nécessite une constante répétition et citation pour être effective.

3 Et c'est précisément parce que les normes de genre ont ce constant besoin d'être citées et répétées qu'elles en deviennent fragiles. Butler parle d'un échec constitutif du performatif dans la mesure où la norme produit dans le même temps une subversion potentielle. Parce que cette "répétition stylisée » est discontinue, son interruption peut permettre de démasquer le processus performatif et de le troubler: cette subversion se déploie par le développement d'une capacité à agir (agency, que l'on peut traduire en français par agentivité) qui repose pour Butler sur la possibilité que chaque réitération puisse en réalité échouer, être réappropriée ou re-signifiée à des fins qui ne seraient pas la consolidation des normes. La construction sociale de la performativité du genre est montrée comme la plus manifeste dans le cas de la drag-queen. Selon Butler, la drag-queen, en performant le genre (en citant et en répétant les énoncés associés au genre féminin), en réalité le subvertit puisqu'elle montre qu'au fond, ce dernier n'est qu'un jeu de rôle. Ainsi, le genre n'est jamais une donnée première, originelle, mais il est bien une imitation, comme l'est la pratique du travestissement. Dans Ces corps qui comptent (Butler, [1993] 2009), Butler souligne cependant la différence entre performance et performativité : la performance quasi théâtrale de la drag-queen présuppose un sujet (puisque la drag-queen sait qu'elle joue un rôle, qu'elle joue à être 
un sujet féminin), tandis que la performativité remet en question la notion même de sujet. La performativité fait ainsi référence à une réalité qui advient à l'intérieur d'un processus de répétition et de citation des normes qui constituent et produisent le sujet, tandis que la performance renvoie à une action effectuée volontairement et consciemment par le sujet. En d'autres termes, si la drag-queen a conscience de sa performance, la plupart des femmes et des hommes (re)construisent incessamment leur identité genrée sans s'en rendre nécessairement compte, ou en intégrant ce processus comme naturel - et donc normal.

4 L'œuvre de Butler évoque principalement les questions du genre et des minorités sexuelles, mais sa pensée relève d'un projet théorique et politique plus étendu : elle permet en effet d'interroger les normes et les discours qui façonnent les corps, afin d'en révéler et d'en subvertir les limites. Prendre pour base la pensée de Butler afin de réfléchir à la conceptualisation d'une performativité raciale (Tate, 2005 ; Ehlers, 2012 ; Byrne, 2000 ; Inda, 2000) nous permettra donc de mieux comprendre la manière dont Ngozi Onwurah place dans ses films les enfants noirs et métis au cœur d'un processus de construction identitaire dans lequel la subversion des normes genrées et raciales reste toujours possible.

\section{Ngozi Onwurah et la figure de l'enfance}

Ngozi Onwurah naît en 1966 au Nigéria, d'un père nigérian noir et d'une mère britannique blanche. Lorsque la guerre civile éclate ${ }^{4}$, elle quitte le Nigéria avec sa mère et son jeune frère pour Newcastle-Upon-Tyne (dans le nord de l'Angleterre), d'où sa mère est originaire (Foster, 1997). Ce changement d'environnement est vécu par la réalisatrice comme un réel traumatisme : "Nous avons quitté le Nigéria à cause de la guerre du Biafra. Puis nous sommes arrivés ici en Angleterre, et avons réalisé que nous entrions dans une autre zone de guerre. Mais nous étions les seuls à nous en rendre compte " (Laballe, 2003). Seuls enfants métis dans une communauté majoritairement blanche, ils deviennent rapidement victimes d'actes racistes à répétition. Ces difficultés d'intégration et d'identification sont au cœur du travail "auto-ethnographique " (Foster, 1997, 34) de la cinéaste, qui réalise son premier court-métrage sur ce sujet (Coffee Coloured Children) en 1988, alors qu'elle est encore étudiante à la prestigieuse Saint Martin's School of Art. Le film remportera plusieurs récompenses et lancera la carrière de la réalisatrice, particulièrement prolifique durant les années 1990. Après plusieurs courts-métrages, elle réalise en 1994 Welcome II the Terrordome, le premier long-métrage de fiction d'une femme noire britannique à être diffusé en salles de cinéma. Elle est toujours active au travers de la société de production qu'elle a fondée avec son frère Simon Onwurah (Non-Aligned Productions) et en juin 2018, tous deux ont été invités à devenir membres de l'Académie des Arts et des Sciences du Cinéma (mieux connue sous le nom d'Académie des Oscars) aux États-Unis.

6 Dans cet article, nous analyserons trois courts-métrages de la cinéaste : Coffee Coloured Children, The Body Beautiful et The Flight of the Swan. Dans ces trois œuvres, la réalisatrice utilise la figure de l'enfance pour dénoncer les systèmes d'oppression psychologiques et physiques qui annihilent les personnes racisées dès la petite enfance. La récurrence de la figure de l'enfance chez Onwurah n'est pas anodine. La réalisatrice a certainement compris que, comme la théorie de l'apprentissage social ${ }^{5}$ l'affirme, l'environnement qui entoure et modèle un enfant le conduit à se conformer aux normes générales de sa 
culture. En montrant ce processus de normalisation comme potentiellement violent, Onwurah brouille les représentations habituelles de l'enfance et bouscule ainsi « des convictions profondément ancrées sur le naturel supposé de l'enfance normative » (Olson; Scahill, 2012). De plus, en évoquant son propre passé, la réalisatrice s'empare d'une pratique autobiographique fondée sur un contrat d'authenticité, qui pourrait permettre une identification plus forte du.de la spectateur.trice avec les personnages de ses films.

\section{Le corps « hors-norme » et ses rituels de subversion : Coffee Coloured Children (1988, 15 minutes, couleur) et The Body Beautiful (1991, 23 minutes, couleur)}

7 Coffee Coloured Children et The Body Beautiful sont deux des premières œuvres de la réalisatrice. Ces deux courts-métrages, largement inspirés de la vie d'Onwurah, évoquent le sentiment de marginalisation que les discours normatifs sur le genre et la « race » peuvent engendrer, notamment chez de jeunes enfants et adolescent.e.s. Ces interrogations autour des politiques identitaires, mais aussi la position centrale qu'occupe le corps "hors-norme » au sein des deux récits, lient indéniablement les deux films (Friedman, 2006). Ces derniers explorent en effet la possibilité pour les corps métis de Ngozi et Simon Onwurah, ainsi que pour le corps handicapé de leur mère (une femme blanche dont la mastectomie et des rhumatismes chroniques ont déformé l'apparence physique), de se transformer en territoire de résistance au travers de différents rituels de subversion.

Coffee Coloured Children, premier court-métrage de la réalisatrice, s'ouvre sur une longue séquence musicale dans laquelle se mêlent à l'écran des photographies d'enfance d'Onwurah et de sa famille, et des images documentaires de la vie quotidienne dans une Grande-Bretagne hétérogène et multiraciale. Le film présente l'image d'une ville " conviviale " (Gilroy 2004) où une multiculture organique et ordinaire se déploie au travers de la cohabitation de personnes de toutes origines sociales, géographiques et ethniques. La chanson enjouée et dynamique ${ }^{6}$ qui accompagne ces images d'ouverture célèbre ce melting pot britannique et la présence au sein de la nation d'enfants « couleur café » (les Coffee Coloured Children du titre du film). Un dessin d'enfant apparaît à l'écran : y sont représentés, souriants et se tenant par la main, une femme blanche, un homme noir et leurs enfants métis (évoquant la réalisatrice et son frère). Ce croquis représentant une famille interraciale heureuse va en réalité à l'encontre des discours racistes de l'époque qui critiquent la « position ambiguë » (Benson, 1981,1) des familles mixtes en Grande-Bretagne ${ }^{7}$. Cette séquence d'ouverture optimiste et fantasmée laisse rapidement place à ce qu'Onwurah présente comme une vision plus réaliste de la situation raciale dans la Grande-Bretagne des années 1970. La scène suivante nous présente un jeune homme blanc étaler sur la porte d'entrée de la famille Onwurah un excrément de chien ramassé dans la rue. Sur la porte souillée, il colle la note suivante : "Monkeys eat shit» (en français : "Les singes mangent de la merde »). Le visage de l'homme n'est jamais montré directement durant cette scène : le film évite ainsi de dépeindre cet évènement comme un fait isolé, mais critique une situation sociale générale où le racisme agit à un niveau individuel. La voix - adulte - de Ngozi commente ce souvenir d'enfance: «Je haïssais cette porte, mais pas autant que je pouvais haïr mon propre reflet dans le miroir ». Les personnages de Ngozi et de son 
jeune frère Simon sont donc initialement présentés comme victimes d'une violence raciste psychologique et matérielle qui conduit à l'internalisation de cette haine raciale. Leur dégoût naît, non seulement de la situation elle-même - du fait que leur porte soit dégradée de la sorte par des motifs racistes -, mais aussi, et peut-être surtout, de ce qu'ils perçoivent être leur inadéquation à être acceptés tels qu'ils sont par ceux et celles qui appartiennent à la dite-norme, c'est-à-dire dans le film par les enfants blancs dont les mères "n'avaient pas à nettoyer leur porte ». L'internalisation du discours raciste provoque donc chez les enfants la connaissance de leur propre corps comme une " activité négatrice » (Fanon, 1952, 92) : ce dernier est perçu comme étranger à soi, et la seule possibilité pour l'enfant de contrer cette dépersonnalisation est de se convaincre, comme le fait la petite Ngozi dans le film, que «quand je serai grande, je serai blanche ».

Le désir naturel de l'enfant de se conformer à la norme - ici la norme de la blanchité (Frankenberg, 1993 ; Dyer, 1997) - s'exprime à travers le déguisement de princesse que revêt un peu plus loin dans le film la petite Ngozi. Plus qu'un déguisement, que de nombreuses petites filles blanches revêtent elles aussi avec plaisir, c'est un réel travestissement qui s'opère chez elle: coiffée d'une perruque blonde et les joues poudrées d'un produit ménager blanchissant, Ngozi joue, en réalité, à être blanche. Car pour l'enfant, être une princesse et être blanche sont bien synonymes : «quand j'étais enfant, je voulais être une princesse, mais je m'attendais à être blanche ». Ce jeu de travestissement est une double performance, puisque ce sont à la fois les normes de la blanchité et du genre féminin qui sont ici récitées et répétées. Mais si cette performance peut rappeler celle de la drag-queen que décrit Butler (tout comme la drag-queen performe le genre féminin, la petite Ngozi performe la blanchité), elle s'en détache en ce sens que la petite Ngozi n'est pas encore dotée de l'agentivité que possède la drag-queen. S'il y a bien un processus conscient de citation de la blanchité (à travers le déguisement), la petite Ngozi n'effectue pas une parodie mais bien ce qu'elle espère être une réelle transformation physique. L'échec de la métamorphose ne peut alors que plonger Ngozi dans une profonde mélancolie que partage son jeune frère Simon, lorsqu'il se voit refuser par le maitre d'école le droit de colorier son visage sur un de ses dessins avec un crayon rose, qui lui semble pourtant la couleur naturelle et normale de sa peau. Le travestissement de Ngozi en princesse semble annuler toute forme de subversion et conduire au contraire à une annihilation encore plus profonde. Cet événement montre la réalité de la performativité raciale qui construit le corps de Ngozi en négation : quoi qu'elle fasse, Ngozi n'est pas (acceptée comme étant) blanche. Ce refus de performer l'identité raciale qui lui a été assignée provoque un effet de dissonance que l'on retrouve chez le jeune Simon à travers son problème d'identification à la couleur du crayon brun. Cette inadéquation conduit à l'internalisation d'un discours raciste qui provoque une haine de soi : "mon enfance s'est perdue dans un brouillard de dégoût", déclarent tristement les deux protagonistes dans le film.

10 L'idée d'une performance vouée à l'échec se retrouve d'une manière toute différente dans The Body Beautiful, dans lequel une Ngozi cette fois adolescente tente, en pressant sa poitrine contre son torse, de se glisser dans la peau de sa mère. Cette performance d'un corps marqué par la mastectomie ne peut qu'échouer car sur le propre aveu du personnage : "C'était inutile, comme fermer ses yeux pour essayer de savoir ce que cela fait d'être aveugle, avant de les ouvrir à nouveau ». Si Ngozi s'empare ici de manière consciente des caractéristiques du corps déformé de sa mère, c'est en partie pour se 
rapprocher physiquement de celle dans le reflet de laquelle elle avoue ne pas se reconnaître. Tout comme la petite Ngozi déguisée en princesse dans Coffee Coloured Children, la Ngozi adolescente de The Body Beautiful tente donc également de s'assimiler à la norme de la blanchité, de laquelle son métissage ne peut que l'éloigner. Dans The Body Beautiful, la réalisatrice s'interroge ainsi sur les difficultés d'identification que les enfants métis peuvent rencontrer par rapport à leur mère blanche. Une des premières séquences du film nous montre la petite Ngozi et son frère Simon en train de jouer sur un carrousel en compagnie de deux petits garçons blancs, tandis que leur mère les surveille tendrement un peu plus loin. Cette scène, a priori anodine, souligne avec subtilité que les difficultés d'identification avec une mère "phénotypiquement" différente peuvent subsister au quotidien. La chercheuse Diana Adesola Mafe espère que « la première réaction du spectateur en voyant cette scène sera de reconnaître les liens familiaux et l'évident lien maternel entre Madge et ses enfants, plutôt que de simplement y voir une femme blanche assise sur un banc et deux enfants noirs jouant plus loin sur un manège » (Mafe, 2008). Par cette remarque, Mafe souligne la manière dont Onwurah confronte les spectateur.trice.s avec leurs possibles attentes et préconceptions, la réalisatrice brouillant même davantage les pistes en montrant deux enfants blancs jouer sous le regard de Madge. Ce qui est représenté ici de manière implicite est en revanche verbalisé par Simon dans Coffee Coloured Children. Simon adolescent relate en effet que durant son enfance, il était régulièrement confronté à des personnes qui ne voulaient pas croire que cette femme blanche était bien sa mère, ajoutant que " quelques fois, juste quelques fois, je pensais que peut-être, elle ne l'était pas ». Ce désir de prouver sa filiation à une mère dont la couleur de la peau est différente de la sienne passe dans les deux films par une performance d'imitation à la blanchité, qui sous-tend la présence d'une souffrance à la fois physique et psychologique. Mais en filigrane se dessine également un discours sur le caractère hybride que revêt l'identité métisse : la sociologue britannique Shirley Tate souligne en effet qu'au cours de leur construction identitaire, les enfants métis doivent également " constamment prouver" (Tate, 2005, 1) leur authenticité et leur appartenance à la blackness. Selon l'auteure, « on a cette idée que la connaissance que l'on a de sa propre identité est basée sur la teinte de sa peau. Plus elle est foncée, plus on est supposément conscient de son identité Noire » (Tate, 2005, 1). Dans Coffee Coloured Children et The Body Beautiful, la figure paternelle est absente : l'impossibilité d'une identification à un père noir contribue donc potentiellement à entraver ce processus de construction identitaire ${ }^{8}$, puisque la blanchité reste l'unique point de référence que les enfants cherchent dès lors à imiter.

Le penseur franco-tunisien Albert Memmi propose une définition de l'imitation dans le contexte colonial qui peut faire écho aux deux courts-métrages d'Onwurah: Memmi observe que le processus mimétique employé par le colonisé pour se rapprocher du colonisateur rend problématique les possibilités de subversion dans le sens où le « souci appliqué de se confondre avec le colonisateur, de s'habiller comme lui, jusque dans ses tics et sa manière de faire la cour » (Memmi, 1957, 140-141) mène à l'échec inévitable du colonisé à s'assimiler au colonisateur. Memmi nous montre que les tentatives d'assimilation du colonisé n'ont aucune valeur tant qu'elles ne sont pas approuvées par le colonisateur : dans le cas contraire, l'imitation deviendra au mieux une "singerie", le colonisé « un singe » (Memmi, 1957, 141). Contrairement à la performance de la dragqueen qui ne cherche pas l'approbation de la gent féminine pour subvertir le genre avec succès, l'imitation ne peut ici être réussie sans l'approbation de celui ou celle qui 
est imité.e. Comme Simon l'avoue dans Coffee Coloured Children, malgré les efforts des deux enfants pour s'approprier les codes de la blanchité (en portant une perruque blonde, en poudrant leurs joues, en utilisant un crayon rose pour colorier leur visage, ou plus loin dans le film en utilisant des produits ménagers chimiques dans l'espoir de blanchir physiquement leur peau), leur imitation est un échec puisque le lien de filiation entre eux et leur mère blanche n'est pas systématiquement reconnu. L'approche phénoménologique de Memmi nous montre qu'en "changeant de peau " (Memmi, 1957, 137), le colonisé refuse sa propre image : « le refus de soi et l'amour de l'autre sont communs à tout candidat à l'assimilation [...] L'amour du colonisateur est sous-tendu d'un complexe de sentiments qui vont de la honte à la haine de soi " (Memmi, 1957, 138). C'est bien cette « haine de soi » qui est évoquée dans Coffee Coloured Children, dans lequel l'intégration du discours raciste contribue à « carencer » (Memmi, $1957,134)$ un sujet construit comme " hors-norme ». Les personnages se mesurent dès lors à leur incapacité à atteindre la norme de la blanchité, et l'échec de l'imitation contribue à forger les contours d'une identité raciale en négatif de cette norme. Dans le film, la voix de Ngozi adolescente confie que « les robes et le maquillage sont devenus de plus en plus ridicules, l'automutilation de plus en plus précise ». Plus précise et plus violente physiquement: pour accélérer le processus de transformation, nous voyons son personnage s'asperger de Domestos, un produit ménager composé d'eau de javel. Simon - enfant, puis adolescent - est quant à lui montré dans son bain, en train de frotter sa peau à l'aide d'une brosse et d'une poudre ménagère blanchissante (" pour faire partir le noir »). Sa voix adulte nous explique qu'il pourra ainsi accéder à la norme et prouver enfin le lien qui l'unit à sa mère. Cette recherche d'un "sérum de dénégrification" (Fanon, 1952, 89) souligne l'idée d'une blackness « construite comme un manque/un échec/une pathologie » (Tate, 2016) puisque les enfants perçoivent leur identité raciale comme une maladie, qui pourrait être potentiellement soignée à l'aide de diverses stratégies.

La mise en scène de ces scènes de bain se fait à travers une représentation dite haptique du souvenir d'enfance qui «fait appel à la mémoire [...] à travers le recours à une connaissance non visuelle, d'une connaissance incarnée, et de l'expérience des sens, tels que le toucher, l'odorat et le goût " (Marks, 2000, 2). L'engagement du.de la spectateur.trice avec cette esthétique haptique se fait non seulement à travers un registre psychique mais également sensoriel: le regard fonctionne alors comme un organe du toucher, laissant supposer "que l'œil lui-même puisse avoir cette fonction qui n'est pas optique » (Deleuze et Guattari, 1980, 614). Les scènes de bain sont filmées dans des formats (super 8 et $16 \mathrm{~mm}$ ) qui donnent une texture granuleuse à l'image. Onwurah joue également d'effets de lumière et de couleurs : le personnage de Simon adolescent apparaît au travers d'un filtre bleu-gris, tandis qu'un faisceau de lumière s'attarde ponctuellement sur son corps, mettant en exergue la brutalité de son geste lorsqu'il frotte violemment sa peau. Mais la performance qui est ici ostensiblement mise en scène est une fois de plus vouée à l'échec, puisqu'elle n'apporte qu'une annihilation psychologique plus profonde et une douleur physique indéniable (Simon avoue en effet que pour faire partir le noir de sa peau, il allait jusqu'à la frotter «jusqu'au sang»). L'utilisation d'un filtre coloré gommant de l'image toute couleur vive rappelle que le monde dans lequel vivent les deux enfants «ne voit qu'en noir et blanc", comme se lamentera plus tard Ngozi dans The Body Beautiful. Le son désynchronisé de la brosse frottant contre la peau de Simon accompagne ces images, visant vraisemblablement à provoquer chez le.la spectateur.trice un sentiment 
d'inconfort, alors que le rythme du montage s'accélère et que la caméra se fait de plus en plus proche du corps. Les gestes se font également de plus en plus rapides, et une journaliste s'inquiète en voyant la scène qu' "ils frottent si vite, si fort, que je m'attends à tout moment à voir apparaître du sang " (Seriki, 2018). Certains plans rapprochés donnent l'impression que l'œil vient frôler les peaux : la visualité haptique tire dès lors parti de « la capacité de la caméra d'effleurer plutôt que de montrer les surfaces, les textures et les espaces - de nous rapprocher des choses en provoquant le souvenir sensuel du toucher ou du mouvement " (Deleuze et Guattari, 1980, 162). Le recours à une esthétique haptique permet en outre à la réalisatrice de souligner que l'internalisation du discours raciste se fait également de manière physique, corporelle et sensorielle.

13 Mais le rituel du bain, initialement dépeint dans le film comme un moment de honte lorsque Simon réalise que, malgré ses efforts, sa peau reste indéniablement brune, est montrée à la fin du film comme un moment de re-stylisation : nous voyons tour à tour Simon et Ngozi, adultes, se baigner nus - Simon dans l'océan et Ngozi sous une cascade naturelle. Ce rituel du bain en pleine nature, où cette fois les couleurs vives éclatent à l'écran, offre donc un contraste avec les bains humiliants du début du film en cela qu'il est désormais vu comme purifiant, revitalisant, et permet une seconde naissance du corps. Si l'eau de javel dont Ngozi s'asperge dans le film «ne nettoyait pas ma peau parce que ma peau n'était pas sale », en revanche l'eau naturelle apporte " une réelle propreté, fraîche, pétillante...». Cette distinction soutient l'idée que c'est le corps culturel, celui qui est incessamment stylisé, subjectivé, normalisé socialement qui devient une prison pour Ngozi et Simon, tandis que le retour au corps "naturel» permet la subversion de la stylisation du corps, et ce vers la création de nouveaux discours. Visuellement, ce renversement se traduit par une libération du cadre filmique, puisque les frôlements de la caméra laissent désormais place à des plans plus larges, qui accordent davantage d'espace aux protagonistes. Le rituel libérateur du bain permet à Simon et Ngozi la réappropriation de leur identité raciale et le renversement du processus d'internalisation du discours raciste.

Si «le travail de dés-identification est indispensable à l'émergence de nouvelles identifications et que la honte fait partie intégrante d'un tel phénomène de désidentification » (Tate, 2009, 95), l'humiliation imposée sur les corps des deux enfants permet l'émergence, notamment à travers le rituel du bain, de nouvelles possibilités d'identification. Ce rituel re-signifiant apparaitt également dans le court-métrage The Body Beautiful. Au début du film, une courte scène nous présente en effet Simon (enfant) laver les cheveux de sa mère, offrant ici encore une représentation à contre-courant de la violence des premières scènes de bain de Coffee Coloured Children. Dans The Body Beautiful, les "inadéquations " aux normes genrées et raciales des corps de Simon - à travers son métissage - et de sa mère, Madge - à travers son corps déformé par une mastectomie - s'annulent face au lien d'amour que peuvent se porter mutuellement mère et enfant. L'espace privé de la salle de bains est cette fois présenté comme un espace de protection face au regard normatif de l'extérieur. Car comme le souligne la sociologue Gargi Bhattacharrya, l'espace privé « échappe au scopique et fait revivre la peau comme la bordure sensible et sensuelle de la chaire. Dans cet espace on espère que nos corps seront considérés avec soin, pas inspectés " (Bhattacharrya, 2001, 45). Cette relation de "soin", parfaitement représentée dans cette scène, permet la création de nouvelles significations produites par le corps «marginal» lorsque celui-ci est restylisé : les pratiques quotidiennes qui forment les identités raciales et genrées (par 
exemple pour Simon de frotter sa peau pour en effacer la couleur, comme on l'a vu dans Coffee Coloured Children, ou pour Madge de créer un ersatz de sein avec un mouchoir pour remplacer celui enlevé par la mastectomie, comme le lui conseille son chirurgien dans le film) sont ici subverties. En échappant à "l'invasif régime scopique » (Bhattacharrya, 2001, 45) du regard extérieur, le corps défie momentanément les normes raciales et genrées puisqu'il ne se trouve plus dans la nécessité de les consolider. Dans The Body Beautiful, Onwurah montre les corps dénudés de la mère et de son enfant sans qu'aucun effet visuel ou sonore ne vienne dénaturer la représentation et la caméra se tient dans l'entrebâillement de la porte, laissant le.la spectateur.trice observer la scène avec distance et pudeur. Le savon n'est plus un outil de normalisation comme dans Coffee Coloured Children, mais symbolise la douceur et la pureté qui émane de la relation entre les deux personnages. Lorsque la caméra vient se poser sur le visage de Madge, c'est pour montrer la complicité qui lie la mère et son petit garçon, qui rince tendrement ses cheveux. De nouvelles inscriptions s'apposent alors métaphoriquement sur les corps relâchés de Madge et de Simon: dans l'espace privé de la salle de bains, ces derniers ne sont plus dans l'obligation de se soumettre à une "répétition stylisée " des normes et le processus performatif qui construit les identités raciales et genrées se trouve ainsi troublé. Cet espace «louangé » (Bachelard, 1961, 17) qu'est la maison de l'enfance devient dans la représentation du souvenir un lieu de protection, d'intimité et de bien-être : "avant d'être "jeté au monde" comme le professent les métaphysiques rapides, l'homme est déposé dans le berceau de la maison » (Bachelard, 1961, 26). Cette nouvelle scène de bain est l'illustration du souvenir d'enfance comme d'un lieu privilégié et heureux, et nuance les cruelles représentations du premier court-métrage de la réalisatrice qui présentait alors la maison de l'enfance comme un espace de vulnérabilité. The Body Beautiful montre que si tous les corps sont produits dans et par les discours normatifs, la performativité raciale les positionne cependant différemment : le corps de Madge est ainsi défini par des critères extérieurs en termes de genre, d'âge et de validisme, mais son appartenance raciale est vue comme nonproblématique. À l'inverse, les corps métis de Ngozi et Simon «se situe[nt] dans un contexte raciste où le corps noir continue d'être isolé au sein des relations sociales " (Ehlers, 2012, 10). Mais la nudité qu'arborent les personnages dans les deux films devient chez Onwurah un élément rhétorique important et montre le corps métis et/ou féminin métaphoriquement déshabillé du costume normatif que le regard extérieur l'oblige à porter au sein de la société dominante.

15 Un second rituel subversif qui n'apparaît pas dans The Body Beautiful mais est exercé dans Coffee Coloured Children est le rituel du feu. Dans la scène de clôture du film, Simon et Ngozi sont en effet montrés en train de jeter perruque, robe de princesse et produits blanchissants au cœur d'un bûcher improvisé. La scène semble se dérouler sur le toit d'un immeuble : si le corps naturel est célébré à travers le rituel du bain purifiant, la destruction des instruments de construction sociale du corps racisé se passe bien en cadre urbain, dans l'espace culturel/social. Le feu peut être perçu à la fois comme un symbole de purification et un instrument de justice. C'est certainement cette deuxième dimension qu'il prend à la fin de Coffee Coloured Children, où un sentiment de justice faite prévaut lorsque le bûcher s'éteint enfin. C'est l'étape finale de guérison du processus de re-stylisation des deux enfants: «d'abord frotter, ensuite s'excuser, enfin se battre pour notre peau » explique Simon. Lors de ce second rituel, les deux personnages sont entièrement vêtus de noir, un habit matériel et métaphorique qui contraste avec l'omniprésence du blanc dont ils se couvraient durant la première partie du film (le 
blanc de la robe de princesse ou le blanc de la poudre ménagère). C'est donc en brûlant ces objets, symboles de leur oppression, que Ngozi et Simon se réapproprient en partie leur blackness. Ngozi confesse : «Je suis forte désormais, parce que personne ne pourra jamais me faire autant de mal que j'ai pu m'en faire moi-même ». Sur ces images de bûcher sacrificiel se superposent les performances de Simon et Ngozi enfants: l'enfance gâchée est elle aussi symboliquement jetée au feu, et le passage vers l'âge adulte est présenté comme un processus d'acceptation et de réconciliation. Le film nous montre en effet que cette dernière étape de lutte peut être porteuse d'espoir : se battre pour sa peau nécessite de subvertir les normes raciales imposées sur le corps, et de réaffirmer la couleur de sa peau. Ngozi déclare ainsi que «l'amour de ma vie sera peutêtre blanc, mais le père de mon enfant sera noir ", refusant ainsi un principe de " purification » attendu des femmes métisses en situation coloniale où le choix d'un père blanc permettait d'élever la lignée familiale sur l'échelle de la pigmentocratie ${ }^{9} \mathrm{en}$ blanchissant graduellement la descendance. L'ultime plan du film montre Simon embrasser sa mère, dans un geste de réconciliation et d'union qui transcende les commentaires négatifs qui pourraient dissocier mère et fils à cause de leurs différences raciales.

16 The Body Beautiful se clôture également par une scène d'étreinte entre le personnage adolescent de Ngozi et de sa mère. Madge Onwurah, qui joue son propre rôle dans chacun des deux films, incarne cette blanchité que les deux personnages embrassent (de manière littérale et métaphorique) et la réalisatrice affirme ici son désir de ne pas enfermer ses personnages dans un archétype du.de la "mulâtre tragique" (tragic mulatto) qui « pour toujours se cherche une identité car il ou elle ne se sent ni noir.e ni blanc.he » (Friedman, 2006, 144). Ces scènes d'intimité entre mère et enfants soulignent une fois de plus l'agentivité des personnages: ces derniers se réapproprient la possibilité de créer les contours de leur propre identité, plutôt que de laisser un regard extérieur les définir en négation des discours normatifs sur le genre et la "race ». Les stratégies de réconciliation déployées dans ces deux courts-métrages permettent de renverser les effets psychiques (déni identitaire, haine de soi...) et corporels (violence physique, travestissement) de la stylisation raciale du corps et amènent à l'acceptation des adolescents de leur identité métisse.

\section{La danse comme pratique de résistance : The Flight of The Swan (1992, 12 minutes, couleur)}

17 Lorsque Ngozi Onwurah réalise en 1992 The Flight of the Swan, elle décide cette fois de ne pas puiser directement dans des souvenirs autobiographiques ou de mettre en scène des personnages qui pourraient évoquer des membres de sa famille. En revanche, son héroïne, une petite fille nigériane qui quitte son village natal pour une GrandeBretagne dépeinte comme froide et hostile, fait indirectement écho à son propre parcours. À Londres, cette petite fille dénommée Obe découvre le monde du ballet et, subjuguée par la grâce et la beauté des danseuses, décide de suivre une formation pour devenir un jour danseuse étoile. Son ambition est cependant rapidement étouffée par un milieu disciplinaire montré comme institutionnellement raciste et par $\mathrm{d}$ es camarades de classe qui se moquent ouvertement d'elle: "Vous imaginez un cygne noir ? Tout le monde pensera qu'elle est tombée dans un tas de charbon !». Le film s'ouvre sur une rencontre quasi mystique entre la jeune héroïne et un personnage féminin imaginaire. Alors qu'elle se promène dans un village africain que la réalisatrice 
n'identifie pas précisément, Obe se fait mordre par un serpent et s'évanouit sur le sol. Rapidement, une femme qui n'est pas sans rappeler la figure de la déesse Aja, célébrée au Nigéria pour sa sagesse et son pouvoir de guérison à la fois physique et spirituelle (Auset, 2009), apparaît et sauve la petite fille. Mais sans que ce changement de décor ne soit expliqué, cet environnement fantasmé, protecteur et bienveillant est bientôt remplacé par celui plus strict du cours de ballet, où la présence d'Obe est perçue comme incongrue. Alors qu'elle se présente à une audition dans le but d'intégrer une prestigieuse école de danse, une petite fille blonde glisse à son insu une poupée de chiffon noire dans son sac. La poupée évoque l'instrument d'un rituel vaudou et l'acte est dépeint comme clairement malicieux : à sa vue, la jeune Obe éclate en sanglots et nous découvrons bientôt que son audition n'a pas été concluante.

Dans la scène finale du film, Obe, les cheveux retenus en chignon, vêtue d'un tutu de danseuse et le visage fardé de blanc (le blanc de la danseuse étoile, mais aussi le blanc symbolique de la blanchité), se glisse à pas feutrés sur la scène d'une salle de spectacle. Sur la célèbre mélodie du Lac des Cygnes, Obe se lance dans une série de pas de danse classique mais rapidement ses mouvements l'étourdissent, et la petite fille s'écroule au sol. Si la déesse protectrice ne vient cette fois pas la sauver, la petite fille perçoit malgré tout sa présence et la remarque recroquevillée dans un coin de la scène. Lorsqu'elle l'aide à se relever, cette dernière efface de ses mains le fard blanc qui recouvre les joues et le front d'Obe, révélant sous le maquillage son vrai visage. Débute alors une danse hybride entre les deux personnages, mêlant mouvements classiques et danse africaine. Sourire aux lèvres, Obe investit l'espace avec confiance et semble à sa place pour la première fois depuis son arrivée en Grande-Bretagne. Onwurah présente la femme et l'enfant au sein d'un décor à la lumière et aux couleurs changeantes, accentuant l'aspect dramatique de la scène. Mais la performance n'est pas destinée à un public extérieur : la salle de spectacle vide symbolise l'importance pour l'enfant (déjà évoquée dans Coffee Coloured Children et The Body Beautiful) de construire son identité à l'écart des regards scrutateurs de la société dominante. Tout comme The Body Beautiful montre l'espace physique de la salle de bains comme un lieu de relâche où le corps "horsnorme " peut retrouver son pouvoir d'agir (agency), la salle vide de The Flight of the Swan se transforme en un espace d'empowerment où la danse, sous cette nouvelle forme hybride, devient une pratique de résistance et non plus d'assimilation aux normes genrées et raciales. Si une partie de la scène est filmée en plan large, la caméra suit également les personnages de près, offrant occasionnellement un point de vue subjectif sur certains mouvements de la déesse, et se plaçant ainsi au cœur du discours qui est en train de se créer (et non pas, une fois de plus, depuis un point de vue extérieur). Le dernier plan s'attarde sur les silhouettes des deux personnages : la déesse soulève Obe et celle-ci tend les bras vers le ciel. L'envol du cygne évoqué dans le titre est donc complet, car c'est en embrassant ses origines et la couleur de sa peau que la petite fille pourra, selon Onwurah, réellement construire son identité au sein d'une société dominante blanche.

En laissant entendre que le refus de l'école de danse à accepter la candidature d'obe pourrait être motivé par des raisons racistes, le film souligne que, tout comme la princesse, la danseuse étoile n'existe dans l'imaginaire populaire que comme blanche. Il est intéressant de noter que dans Coffee Coloured Children et The Body Beautiful, Gwendolyn Audrey Foster (Foster, 1997) identifie le motif du voile comme un élément central : le tissu vaporeux de la robe de princesse que Ngozi finit par brûler à la fin du 
premier court-métrage est mis en parallèle avec le tissu qu'elle laisse tomber de ses épaules dans The Body Beautiful lorsqu'elle se tient face à son miroir et tente d'imaginer son corps privé d'une poitrine. Dans The Flight of the Swan, l'image du voile est également présente au travers du vêtement de danse, symbolisant non seulement la blanchité, mais également la quintessence d'une féminité associée à la grâce et la pureté. Si la performance de Ngozi dans le voile de la princesse mène à un échec, Obe subvertit dans son tutu de danseuse les codes qui sont associés à la norme de la blanchité en les parodiant, et déploie ainsi son agentivité en investissant un nouvel espace hybride. En s'emparant de la figure de la danseuse étoile, incarnation de la féminité dans l'imaginaire populaire, elle contrecarre également un "système impérialiste qui désignait les femmes noires comme non féminines " (Whelehan, 1995, 116). Si Obe ne brûle pas le voile symbolique de la blanchité, elle lui appose une nouvelle signification. Cette performance se rapproche davantage de la définition l'imitation (mimicry) qu'Homi K. Bhabha propose dans Les lieux de la culture (Bhabha, 2007). Bhabha envisage les phénomènes d'imitation en situation coloniale de manière quelque peu différente que ne le fait Albert Memmi. S'inspirant (tout comme Butler) de la définition du performatif de J.L. Austin, il perçoit en effet l'imitation dans sa dimension subversive, car "loin de reproduire l'image projetée par le colonisateur, [elle] implique une "représentation partielle", forcément ironique et parodique " (Bhabha, 1984, 125-133). L'imitation expose ainsi en réalité l'artificialité du discours colonial. Mais le terme mimicry chez Bhabha va en réalité au-delà de l'idée d'une simple imitation: il dénote plutôt un processus de camouflage qui place le sujet dans une position d'ambiguïté où il est " presque le même mais pas tout à fait » que celui qu'il imite. Bien que ce processus de camouflage sous-tende un certain effacement de la culture d'origine au profit de la culture d'adoption, l'espace d'hybridité qui se déploie dans la séquence finale de The Flight of the Swan laisse apparaittre les possibilités de subversion et d'adaptation que l'imitation en tant que parodie apporte.

La thématique de la danse constitue par ailleurs un choix intéressant pour montrer ce processus d'imitation : en effet, pour le philosophe Joshua Hall, « la danse [...] possède d'importantes ressemblances phénoménologiques avec la description que Fanon fait du corps Noir dans les cultures racistes et coloniales » (Hall, 2011). Dans Les Damnés de la Terre, Fanon décrit la danse comme un processus de libération durant lequel le colonisé peut « s'exorciser, s'affranchir, se dire » (Fanon, 2002, 59) et contrer la conscience de son corps en "triple personne" (Fanon, 1952, 155). Cette conscience en "triple personne " provoque une "angoisse raciale» (Marriott, 2007, 208) et crée une stylisation du corps - « un décollement, un arrachement, une hémorragie qui caillait du sang noir sur tout mon corps » (Fanon, 1952, 155) - qui pourrait être re-signifiée à travers la danse. Hall émet ainsi l'hypothèse suivante :

Peut-être la prise de conscience acquise par les personnes Noires dans leur expérience de l'oppression Blanche a-t-elle augmenté leur capacité à percevoir les mouvements humains depuis différentes perspectives, leur permettant alors d'acquérir des informations potentiellement précieuses dans la société, et largement invisibles aux personnes Blanches qui les oppressent. (Hall, 2011).

21 Hall compare ainsi la prise de conscience de son corps par le danseur, encouragé par l'abondance de miroirs qui tapissent les salles d'entraînement, à celle de la personne noire qui grandit dans une société majoritairement blanche et qui prend conscience de son corps à travers le regard extérieur blanc. Pour Yvon Fotia enfin, la danse est « une dialectique de la matérialisation, elle permet de refuser la disparition totale de l'être, la 
fin du colonisé, sa soumission, son aliénation et sa confirmation totale à l'ordre colonial» (Fotia, 2009). Cette expression du corps, telle qu'elle est réinvestie par Obe dans The Flight of the Swan, permet donc à la petite fille de « refuser la disparition totale » de son essence profonde, entamée quelques scènes plus tôt lorsqu'elle détruit matériellement la poupée de chiffon noire. Tout comme les enfants représentés dans Coffee Coloured Children et The Body Beautiful, Obe construit bien son identité raciale au travers initialement d'un certain processus d'imitation, avant qu'il ne se transforme en processus de subversion et enfin d'acceptation de son identité.

\section{Performativité raciale et possibilités de subversion}

Ces trois courts-métrages montrent que, comme l'identité genrée, l'identité raciale n'est pas quelque chose de fixe et d'immuable qui existe dès la naissance et ne se modifie plus par la suite, mais qu'elle se construit à travers une série d'actes performatifs. C'est bien l'incorporation des normes sociales (ce qu'une personne racisée est censée faire ou ne pas faire dans la société) qui construit l'identité raciale, au travers d'une série d'actes discursifs et corporels qui stylisent le corps. Comme la réalisatrice le rappelle régulièrement dans ses films ${ }^{10}$, la construction sociale du corps noir selon certaines caractéristiques stéréotypées prétendument naturelles (comme paresseux, primitif, ou encore hypersexuel) est héritée de l'époque de l'esclavage et du colonialisme. Le racisme se traduisait alors par une stylisation raciale du corps noir passant par une série d'actes discursifs et gestuels :

Il semblait naturel, du moins selon la perspective des propriétaires d'esclaves, que les femmes "blanches» montent à cheval et que les esclaves masculins leur courent après pour les abriter du soleil avec une ombrelle; que les hommes «blancs» s'assoient et que les esclaves restent debout; que les surveillants «blancs» examinent les femmes esclaves comme des trophées de chasse, ou punissent les esclaves en fugue avec des formes banales de torture. (Inda, 2000)

Selon cette division des rôles, on n'effectue dès lors pas les mêmes tâches physiques selon son genre et son appartenance raciale. En revanche, nos identités se construisent à travers certains actes performatifs : elles en sont la conséquence et non la cause. Les femmes blanches ne sont ainsi pas nées pour être des cavalières et les hommes noirs pour les ombrager: ces comportements ne sont pas naturels mais construits socialement. C'est donc bien l'incorporation des normes sociales qui construit l'identité raciale puisque «si le sujet noir et l'expérience noire ne sont pas stabilisés par la Nature ou par quelque autre garantie essentielle, alors ils doivent nécessairement être construits historiquement, culturellement et politiquement » (Hall, 2017, 407).

Il n'y a en effet pas de raisons sensées qu'Obe dans The Flight of the Swan ne puisse pas tenter de devenir une danseuse étoile, puisqu'il s'agit en grande partie d'une pratique artistique dont on acquiert la connaissance après de longues années de formation et non pas un talent inné lié à l'identité raciale d'une personne. Mais lorsque la petite fille découvre la poupée noire glissée malicieusement dans son sac, la perception, en « triple personne ", qu'elle fait de sa propre existence se conforme dès lors à ce postulat normatif qu'une petite fille noire ne puisse pas être danseuse de ballet. Elle construit ainsi, à son arrivée en Grande-Bretagne, son identité noire comme quelque chose de négatif, puisque le discours raciste auquel elle est confrontée semble ne lui laisser aucune autre possibilité. Cette scène révèle la douloureuse expérience que cette connaissance en «triple personne » provoque chez l'enfant racisé : Obe, la poupée à la 
main, ne remarque tout d'abord pas un groupe de danseuses plus âgées qui s'entraîne plus loin dans une salle de classe. À l'avant-plan se détache le personnage de la déesse, vêtue cette fois d'un tutu blanc et les cheveux coiffés de rubans de la même couleur. La réalisatrice crée un lien entre la poupée et la danseuse : alors qu'une Obe sanglotante détruit et piétine la poupée de chiffon, la déesse tombe au sol et se tord de douleur. C'est ici son identité noire qu'obe tente de détruire, puisqu'elle est le principal obstacle à la réalisation de son désir de devenir danseuse. Les deux personnages se toisent du regard - mais en réalité, en demandant à ses actrices de fixer directement et fermement l'objectif de la caméra, c'est à nouveau le.la spectateur.trice qu'Onwurah interpelle.

Coffee Coloured Children, The Body Beautiful et The Flight of the Swan montrent que les actes de racisme commis par autrui (la poupée de chiffon glissée dans le sac d'obe ou encore la crotte de chien étalée sur la porte de la famille Onwurah) s'accompagnent d'une internalisation chez les personnes racisées de cette négation de leur propre existence, un phénomène aliénant et destructeur que la réalisatrice dénonce. L'effet de dissonance dont ses personnages font l'expérience (entre la perception de leur propre identité et l'image que la société dominante blanche leur renvoie) laisse transparaître l'influence marquante de la pensée de Fanon sur l'œuvre de la réalisatrice (Hladki, 1995 ; Kauffman, 1998 ; Foster, 1997). Pour le philosophe et psychiatre martiniquais, «le colonisé finit par intégrer ces discours de stigmatisation, le sentiment d'être inférieur, il finit par mépriser sa culture, sa langue, son peuple, il ne veut plus alors qu'imiter, ressembler au colonisateur" (Canonne, 2016). Mettant en lumière la continuité historique des effets dévastateurs de l'esclavage et du colonialisme sur l'expérience contemporaine de la diaspora noire (Varaidzo, 2017), les films d'Onwurah montrent que cette dernière reste, à l'ère postcoloniale, victime de procédés racistes similaires à ceux que Fanon dénonce dans les années 1950 et 1960 . Ce sont les discours de stigmatisation (à travers le refus du maître de laisser Simon colorier son visage en rose ou de l'école de ballet d'accepter la candidature de la jeune Obe) et la stylisation raciale de leurs corps (à travers un processus de blanchiment de leur peau pour Simon, Ngozi et Obe) qui poussent les personnages d'Onwurah à vouloir imiter la norme dominante, malgré la violence psychique et physique que celle-ci engendre. Cette internalisation du discours raciste montre que la performativité raciale permet la perpétuation de systèmes inégalitaires, parfois au travers de l'approbation involontaire des individus dominés :

L'idéologie d'une classe dirigeante, on le sait, se fait adopter dans une large mesure par les classes dirigées. [...] En consentant à cette idéologie, les classes dominées confirment, d'une certaine manière, le rôle qu'on leur a assigné. Ce qui explique, entre autres, la relative stabilité des sociétés ; l'oppression y est, bon gré mal gré, tolérée par les opprimés eux-mêmes. (Memmi, 1957, 108-109)

Parce que l'identité raciale est instaurée par une répétition de ce rôle assigné, la « race » en tant que catégorie sociale devient performative. L'identité raciale ne passe donc pas tant par la couleur de la peau, mais est plutôt générée par un «ensemble de significations attachées à la peau - des significations qui, à travers diverses technologies et techniques, en arrivent à réguler, discipliner, et former les sujets comme racisés » (Ehlers, 2012, 14). Mais si l'identité raciale est «formée », alors tout laisse à penser qu'elle puisse être déformée : c'est précisément ce que les trois courtsmétrages analysés ici démontrent. Dans ces films, cette déformation peut passer par différents procédés d'ordre psychique et corporel. Reconnaissant le pouvoir d'une force extérieure qui «initie et forme» (Dorlin, 2007) le sujet dominé malgré lui, Achille 
Mbembe distingue deux formes d'affranchissement possibles : la "pratique absolue de la violence " (Dorlin, 2007) qu'il identifie chez Fanon, tandis que «chez Butler, il faut aller la trouver dans des actes somme toute abstraits tels que l'auto-reproche, la mélancolie et l'interdit et pas vraiment dans le soulèvement ou encore l'insurrection " (Dorlin, 2007). L'auto-reproche, la mélancolie et l'interdit sont bien des caractéristiques que l'on retrouve chez les personnages d'enfants des films d'Onwurah. Mais si la vie psychique est impliquée dans leur processus d'affranchissement (à travers la prise de conscience qui s'opère en eux à la fin de l'enfance), c'est bien l'affirmation du corps, à travers un processus de dé/re-stylisation, qui permet la performance de la blackness. Cette performance s'apparente à ce que Shirley Tate nomme le phénomène "Peau Noire/Masque Noir» (Tate, 2005), se référant au célèbre ouvrage de Fanon Peaux Noires, Masques blancs (Fanon, 1952). Pour Tate, le processus d'identification - la manière dont on se définit et dont on se nomme - est une pratique qui prend son sens à travers une traduction des discours identitaires. À travers un processus d'identification - à la blackness - et de dés-identification - à la blanchité - le sujet «façonne, stylise, produit et "performe" ces positions [identitaires] » (Tate, 2015, 183). Dès lors, « dire "je suis noir.e" est, dans un seul et même temps, se situer politiquement, socialement, intellectuellement, philosophiquement, culturellement, "racialement", psychiquement et affectivement, peu importe combien être noir.e puisse être contesté » (Tate, 2015, 183). Nous ajouterons que l'identification à la blackness signifie également se positionner physiquement, matériellement. La performativité raciale peut ainsi être resignifiée, ré-inventée, à travers notamment des actes de revalorisation du corps noir en sont l'exemple le mantra Black is Beautiful du Black Power, l'émergence de l'Afroesthétique dans le Brésil des années 1970 ou encore la promotion des peaux foncées et des cheveux naturels par le mouvement Rastafari (Tate, 2015, 183). Ce processus de désidentification (qui fait intégralement partie du rite de passage de l'adolescence dans les films d'Onwurah) est nécessaire à l'émergence de l'agentivité qui sous-tend toute résistance au discours normatif et donne naissance à de nouvelles significations et identifications.

\section{Conclusion}

Le cinéma d'Onwurah est résolument provocateur et radical, et la réalisatrice ne cache aucunement son désir de bousculer son public ou ses critiques : "Ce qu'ils attendent d'une réalisatrice Noire, ce n'est clairement pas le genre de films que moi j'ai envie de faire " (Foster, 1997: 38). Par cette affirmation, elle répond aux controverses qui entourent la plupart de ses films ${ }^{11}$ et refuse d'endosser un rôle de déléguée au nom de la communauté noire. Ses films constituent une œuvre sans concession, un travail qui est cependant «dur à absorber : il est honnête, et son honnêteté est brutale. Le viol est montré comme un viol. La violence est montrée de manière violente. Le racisme est montré comme historique " (Varaidzo, 2017). Dans Coffee Coloured Children, The Body Beautiful et The Flight of the Swan, la figure de l'enfant est montrée comme la première victime des discours racistes, et la violence de certaines images (le frottement de la brosse contre la peau de Simon dans le premier court-métrage de la réalisatrice par exemple) vise à provoquer un sentiment d'inconfort chez le.la spectateur.trice. Onwurah refuse les discours normatifs sur les supposées douceur, innocence et pureté naturelles de l'enfance (Jones, 2015), en démontrant que ces discours sont en réalité fondés sur des notions raciales qui sont spécifiques. C'est bien parce que Ngozi, Simon 
et Obe sont des enfants racisés qu'il leur est impossible, selon la réalisatrice, de construire leur identité de manière non problématique et que leur enfance ne peut donc être ni douce, ni innocente.

Ainsi, ces personnages d'enfants sont sans cesse ramenés à leur impossibilité d'accéder à la norme de la blanchité - c'est-à-dire par exemple à correspondre aux moules établis de la princesse ou de la danseuse étoile. Leur identité raciale se forme en filigrane d'actes performatifs répétés et imposés de manière directe ou indirecte sur les corps comme l'obligation pour Simon Onwurah de colorier son visage en brun à l'école. Si la stylisation raciale du corps, à travers «toutes sortes de gestes, de mouvements et de styles corporels qui donnent l'illusion d'un soi ["racial"] durable» (Butler, 2005, 265), transforme la « race » en un acte performatif, cela signifie qu'un enfant développera bien des capacités et des aptitudes différentes selon son appartenance raciale. Mais la discontinuité de cette répétition stylisée peut permettre de troubler le processus performatif, la réitération peut être donc réappropriée (comme dans The Flight of the Swan) ou re-signifiée (comme dans Coffee Coloured Children) dans un but autre que celle de la consolidation de la norme raciale. Une société normative, qui « ne voit qu'en noir et blanc » selon Ngozi dans The Body Beautiful, offre donc malgré tout des possibilités de résistance qui sont explorées dans le cinéma de la réalisatrice. En effet, si c'est bien l'incorporation des normes sociales, à travers une réitération et une répétition indéfinie d'énoncés récités par les corps qui crée l'identité raciale, les films d'Onwurah montrent que cette dynamique peut être subvertie afin de donner de nouvelles significations à l'identité raciale. Ce travail de dés-identification permet la création de "stratégies de survie que le sujet minorisé utilise afin de négocier son trajet dans la sphère publique majoritaire qui efface ou punit continuellement l'existence de sujets qui ne se conforment pas au fantasme d'une citoyenneté normative» (Munoz, 1999). Troubler la performativité raciale, à travers un ré-investissement et une re-stylisation du corps, permet donc de transformer la performance de la blackness en un acte subversif. Mais si le corps est montré dans le cinéma d'Onwurah comme un outil de contestation, la réalisatrice rappelle que le discours raciste que ses personnages tentent continuellement de contrer se situe à un niveau structurel, institutionnel et historique. En cela, son œuvre s'inscrit dans une volonté politique de dénoncer les réalités du racisme, au-delà de "simplement » créer des représentations positives de la blackness. Peut-être est-ce cela qui permet à son cinéma de rester si actuel, un constat que la réalisatrice fait cependant amèrement lors de la projection de Coffee Coloured Children, The Body Beautiful et The Flight of the Swan lors du London Short Film Festival en janvier 2018 (Seriki, 2018). C'est aussi cela qui fait de Ngozi Onwurah une des réalisatrices les plus singulières de l'histoire du cinéma britannique.

\section{BIBLIOGRAPHIE}

Amadiegwu, Mildred. « Don't shoot this messenger », The Guardian (2006) : https:// www.theguardian.com/media/2006/jul/03/mondaymediasection.race 
Auset, Brandi. The Goddess Guide : Exploring the Attributes and Correspondences of the Divine Feminine. Woodbury (USA) : Llewellyn Publications, 2009.

Bachelard, Gaston. La Poétique de l'Espace. Paris : Les Presses universitaires de France, 1961.

Bandura, Albert. Social Learning Theory. New Jersey : Prentice-Hall, 1977.

Benson, Susan. Ambiguous Ethnicity : Interracial Families in London. Cambridge : Cambridge University Press, 1981.

Bhabha, Homi. "Of Mimicry and Man : the Ambivalence of Colonial Discourse », Discipleship : A Special Issue on Psychoanalysis, 28 (1984) : 125-133.

--- Les lieux de la culture : une théorie postcoloniale. Paris : Payot, 2007.

Bhattacharrya, Gargi. «Flesh and Skin : materialism is doomed to fail », in Contested Bodies. Ed. Ruth Holliday, John Hassard. New York - Londres : Routledge, 2001. 36-47.

Butler, Judith. Trouble dans le genre. Pour un féminisme de la subversion. C. Kraus trans. Paris : Éditions La Découverte (2005) [1990].

--- Ces corps qui comptent. De la matérialité et des limites discursives du « sexe ». C. Nordmann trans. Paris, Amsterdam (2009) [1993].

Byrne, Bridget. « Troubling race. Using Judith Butler's work to think about racialised bodies and selves », présentation au séminaire Queering Development : Challenging Dominant Models of Sexuality in Development, 2000.

Canonne, Justine. «Frantz Fanon : contre le colonialisme », Sciences Humaines, 233 (2016) : 28.

Deleuze, Gilles et Félix Guattari. Mille Plateaux. Paris : Éditions de Minuit, 1980.

Dorlin, Elsa. « Décoloniser les structures psychiques du pouvoir. Érotisme raciste et postcolonie dans la pensée d'Achille Mbembe », Mouvements, 3 : 51 (2007) : 142-151.

Dyer, Richard. White. Londres : Routledge, 1997.

Ehlers, Nadine. Racial Imperatives : Discipline, Performativity \& Struggles Against Subjection.

Bloomington - Indianapolis : Indiana University Press, 2012.

Fanon, Frantz. Peau Noire, Masques Blancs. Paris : Éditions Du Seuil ; 1952.

--- Les damnés de la Terre. Paris : Editions La Découverte, 2002 [1961].

Foster, Gwendolyn. Women Filmmakers of the African \& Asian Diaspora : Decolonizing the Gaze, Locating Subjectivity. Carbondale :Southern Illinois University Press, 1997.

Fotia, Yvon. « Frantz Fanon, la vérité noire, l'expérience anti-coloniale », Les Figures de la Domination (2009) : http://www.lesfiguresdeladomination.org/index.php?id=239.

Frankenberg, Ruth. White Women, Race Matters : The social construction of whiteness, Minneapolis : University of Minnesota Press, 1993.

Friedman, Lester. Fires Were Started : British Cinema and Thatcherism. Londres : Wallflower Press, 2006.

Gilroy, Paul. After Empire : Melancholia Or Convivial Culture ? Londres : Routledge, 2004.

Hall, Joshua. « Revalorized Black Embodiment : Dancing with Fanon », Journal of Black Studies, 43 :3 (2011) : 274-288.

Hall, Stuart. Identités et cultures. Politiques des cultural studies. Édition établie par Maxime Cervulle. Traduit de l'anglais par Christophe Jaquet. Paris : Éditions Amsterdam, 2017. 
Hladki, Janice. « Towards a Politics of Difference in Education : a

Canadian perspective ", International Studies in Sociology of Education, 5 :1 (1995) : 41-56.

hooks, bell. Ne suis-je pas une femme ? Femmes noires et féminisme. Paris : Cambourakis, 2015 [1re éd. : 1982]. Traduit de l'anglais par Olga Potot. Note de la traductrice, p. 165.

Huppatz et al. Identity and Belonging. Londres :Palgrave, 2016.

Inda, Jonathan. «Performativity, Materiality, and the Racial Body », Latino Studies Journal, 11 :3 (2000) : 74-99.

Inda, Jonathan ; Miron, Louis. « Race as a Kind of Speech Act », Cultural Studies, 5, (2000) : 85-107. Jones, Cassandra. « Not the Little Blonde Innocent You Picture : Race and "Innocent" Girlhoods in The Hunger Games Fandom ", in The Child in Post-Apocalyptic Cinema. Ed. Debbie Olson. Londres New York : Lexington Books, 2015. 207-220.

Kauffman, Linda. Bad Girls and Sick Boys : Fantasies in Contemporary Art and Culture, Los Angeles, Londres : University of California Press, 1998.

Laballe, Candace. « Onwurah, Ngozi 1966 », Encyclopedia.com (2003) :

https://www.encyclopedia.com/education/news-wires-white-papers-and-books/onwurahngozi-1966

Mafe, Diana. « Misplaced bodies :probing racial and gender signifiers in Ngozi Onwurah's The Body Beautiful », Frontiers : A Journal of Women's Studies, 29 :1 (2008) : 37-50.

Marks, Laura. The Skin of the Film : Intercultural Cinema, Embodiment and the Senses. Londres : Duke University Press, 2000.

Marriott, David. Haunted Life : Visual Culture and Black Modernity. New Brunswick : Rutdgers University Press, 2007.

Memmi, Albert. Portrait du colonisé, Portrait du colonisateur. Paris : Gallimard, 1957.

Munoz, José Esteban. Disidentifications : Queers of Color and the Performance of Politics. Minneapolis, Londres : University of Minnesota Press, 1999.

Olson, Debbie et Scahill, Andrew. Lost and Othered Children in Contemporary Cinema. Plymouth : Lexington Books, 2012.

Ravi Rampersad, «Interrogating Pigmentocracy. The Intersections of Race and Social Class in the Primary Education of Afro-Trinidadian Boys ", in Intersectionality and "Race" in Education. Ed. Kalwant Bhopal, John Preston. New York - Londres : Routledge, 2012. 57-75.

Seriki, Rashida. « Ngozi Onwurah, Three Short Films », The White Review (2018) : http:// www.thewhitereview.org/reviews/three-short-films-ngozi-onwurah.

Tate, Shirley. Black Skins, Black Masks : Hybridity, dialogism, performativity. Farnham : Ashgate Publishing, 2005.

--- Black Beauty : Aesthetics, Stylization, Politics. New York - Londres : Routledge, 2009

--- «Performativity and "raced" bodies ». In Theories of Race and Ethnicity. Karim Murji, John Solomos. Cambridge : Cambridge University Press, 2015. 180-197.

--- Skin Bleaching in Black Atlantic Zones : Shade Shifters. Londres : Palgrave MacMillan, 2016.

Ugochukwu, Françoise. Biafra, la déchirure : Sur les traces de la guerre civile nigériane de 1967-1970. Paris : L’Harmattan, 2009. 
Varaidzo. « Ngozi Onwurah : the forgotten pioneer of black British film », Gal-Dem (2017) : http:// nu.gal-dem.com/ngozi-onwurah-the-forgotten-pioneer-of-black-british-film

Whelehan, Imelda. Modern Feminist Thought. From the Second Wave to 'Post-Feminism'. New York : New York University Press, 1995.

\section{NOTES}

1. Voir le site officiel du Festival : http://shortfilms.org.uk/events/2018-01-17-ngozi-onwurahshort-films

2. Nous définissons le terme blackness comme «le fait d'être noir, impliquant une certaine réappropriation du terme dans une démarche de fierté, qu'on pourrait traduire par « négrité » ou «négritude» (si ce dernier terme ne se référait pas spécifiquement à un courant de la littérature) » (hooks, 2015).

3. Cette notion de performativité du langage est développée par le philosophe du langage britannique John Langshaw Austin dans son ouvrage How to Do Things with words (1962). Londres : Oxford University Press.

4. La guerre civile au Nigeria (ou guerre du Biafra) se déroule de juillet 1967 à janvier 1970. Elle fait suite à une série de coups d'État et au massacre de plus de 50000 Igbos entre mai et septembre 1966. La guerre est déclarée en 1967 lorsque la région du Biafra, un territoire largement occupé par la communauté Igbo, fait sécession et se renomme «République indépendante du Biafra ». Mais cette nouvelle république, riche en gisements de pétrole, ne sera pas reconnue par les autorités nigérianes qui reprendront de force la région du Biafra le 15 janvier 1970 (Ugochukwu 2009).

5. La théorie de l'apprentissage social, portée par le psychologue canadien Albert Bandura, s'intéresse à la construction identitaire de l'enfant en tant qu'ensemble d'habitudes apprises en réponse à l'environnement social. Le choix d'un comportement se fait pour Bandera en fonction des avantages ou des inconvénients qu'il apporte à l'enfant, qui répond ainsi aux normes fixées par la société. Cette dernière punit ou récompense en fonction du comportement choisi (Bandura 1977). La construction identitaire de l'enfant répond dès lors d'une certaine forme de performativité (bien que Bandura n'utilise pas ce terme), puisqu'il construit de nouvelles habitudes comportementales en observant et en imitant les individus qui l'entourent. La connaissance de sa propre identité se fait dès lors en miroir d'un environnement extérieur normatif.

6. La chanson « Melting Pot» (1969) du groupe britannique Blue Mink est un appel bienveillant à créer un monde où une harmonie raciale, culturelle et religieuse puisse régner. Cependant, le ton assimilationniste de la chanson ainsi que son recours à des termes racistes ("Red Indian Boys", «Yellow Chinkies »...) demeurent problématiques (Huppatz et al. 2016).

7. Susan Benson (Benson, 1981) avance l'idée que bien que la présence de familles mixtes au sein de la population britannique au début des années 1980 soit relativement faible, leur supposée transgression des divisions raciales et ethniques, prégnantes dans le discours populaire de l'époque, contribue à rendre leur position au sein de la société ambigüe. Les enfants issus d'une union mixte sont tout particulièrement confrontés à un flou identitaire, parce qu'un discours essentialiste sur les identités raciales tend à les catégoriser uniquement comme noirs, et non pas métis.

8. Ce sera par ailleurs le sujet d'un autre court-métrage de la réalisatrice : I Bring you Frankincense (1996, 33 minutes, couleur), dans lequel un petit garçon métis élevé par une mère célibataire blanche trouve en la figure du chanteur de soul américain Marvin Gaye un père potentiel. 
9. Le terme de pigmentocratie désigne un système social de privilèges et de discriminations qui confère plus ou moins de pouvoir selon la couleur de sa peau : à la couleur de peau la plus claire correspond un niveau plus élevé sur l'échelle sociale. Le phénomène est également basé sur d'autres caractéristiques phénotypiques (texture du cheveu, forme du nez, des lèvres etc.) et fonctionne à la fois interracialement et intraracialement (Rampersad, 2012).

10. Et tout particulièrement dans les courts-métrages And Still I Rise (1992) et White Men Are Cracking Up (1994), ou encore le long-métrage Welcome II the Terrordome (1994).

11. Particulièrement son premier long-métrage Welcome II the Terrordome (1994) et son téléfilm pour la BBC Shoot the Messenger (2006) (Amadiegwu, 2006).

\section{RÉSUMÉS}

Figure majeure du cinéma Black-British, la réalisatrice Ngozi Onwurah reste largement en marge des études sur le cinéma britannique. Son œuvre, souvent autobiographique, évoque régulièrement la figure de l'enfance et la construction identitaire des enfants noirs et métis au sein de sociétés majoritairement blanches. À travers les concepts de stylisation du corps et de performativité du genre de Judith Butler, ainsi que d'une approche phénoménologique et d'une perspective postcoloniale, nous verrons de quelle manière l'enfance chez Ngozi Onwurah révèle certains processus de subversion des identités raciales et genrées.

Although Ngozi Onwurah is a leading figure of Black British cinema, she still remains largely in the margins of British Film Studies. Her work, which is often autobiographical and offers representations of childhood, questions the identity-building process of black and mixed-race children within white societies. While referring to Judith Butler's concepts of the stylization of the body and gender performativity, and combining phenomenological and postcolonial approaches, I will explore how childhood in Ngozi Onwurah's films actually reveals strategies of subversion of racial and gender identities.

\section{INDEX}

Mots-clés : cinéma britannique, Black-British, enfance, performativité, genre, race Keywords : Black British cinema, representations of childhood, performativity of race and gender

\section{AUTEURS}

\section{ÉMILIE HERBERT}

Doctorante Université de Liège (Belgique)

eherbert@doct.uliege.be 